\title{
Tandem Aldol-Michael reactions in aqueous diethylamine medium: a greener and efficient approach to dimedone-barbituric acid derivatives
}

\author{
Assem Barakat ${ }^{1,2^{*}+}$, Abdullah Mohammed Al-Majid ${ }^{1 \dagger}$, Abdulaziz Moshabab Al-Ghamdi ${ }^{1 \dagger}$, Yahia Nasser Mabkhot $^{1 \dagger}$, \\ Mohammed Rafiq Hussain Siddiqui ${ }^{1+}$, Hazem A Ghabbour ${ }^{3+}$ and Hoong-Kun Fun ${ }^{3+}$
}

\begin{abstract}
Background: Green chemistry is a rapidly developing new field that provides us with a proactive avenue for the sustainable development of future science and technologies. Green chemistry uses highly efficient and environmentally benign synthetic protocols to deliver lifesaving medicines, accelerating lead optimization processes in drug discovery, with reduced unnecessary environmental impact. From this view point, it is desirable to use water instead of organic solvents as a reaction medium, since water is safe, abundant and an environmentally benign solvent.

Results: A convenient one-pot method for the efficient synthesis of the novel Zwitterion derivatives 4a-p via a three-component condensation reaction of barbituric acid derivatives $1 \mathrm{a}, \mathrm{b}$, dimedone 2 , and various aldehydes 3 in the presence of aqueous diethylamine media is described. This new approach is environmentally benign, with clean synthetic procedure, short reaction times and easy work-up procedure which proceeded smoothly to provide excellent yield (88-98\%). The synthesized products were characterized by elemental analysis, IR, MS, NMR and $\mathrm{CHN}$ analysis. The structure of $4 a$ was further confirmed by single crystal $X$-ray diffraction. The compound crystallizes in the orthorhombic space group Pbca with $a=14.6669$ (5) $\AA, b=18.3084$ (6) $\AA, c=19.0294$ (6) $\AA, a=90^{\circ}, \beta=90^{\circ},=90^{\circ}$, $V=5109.9$ (3) $\AA^{3}$, and $Z=8$. The molecules are packed in crystal structure by weak intermolecular $C-H \ldots O$ hydrogen bonding interactions.
\end{abstract}

Conclusions: An environmentally benign Aldol-Michael protocol for the synthesis of dimedone-barbituric derivatives using aqueous diethylamine medium is achieved.

Keywords: Tandem Aldol-Michael reactions, MCRs, Barbituric acid, Aqueous media, Green chemistry, Dimedone, Zwitterions

\section{Background}

Recently, the development of environmentally benign and clean synthetic procedures has become the goal of organic synthesis. Water plays an essential role in life processes and also as a medium for organic reactions $[1,2]$. The use of water as a reaction medium exhibits remarkable benefit because of its high polarity and therefore immiscibility with most organic compounds. Reactions in aqueous

\footnotetext{
* Correspondence: ambarakat@ksu.edu.sa

${ }^{\dagger}$ Equal contributors

'Department of Chemistry, College of Science, King Saud University, P.O. Box 2455, Riyadh 11451, Saudi Arabia

${ }^{2}$ Department of Chemistry, Faculty of Science, Alexandria University,

P.O. Box 426, Ibrahimia, Alexandria 21321, Egypt

Full list of author information is available at the end of the article
}

media are environmentally safe and have less carcinogenic effects with a simple work up procedure which are especially important in industry. Thus, there is a need for developing multicomponent reactions (MCR's) in water, without the use of any harmful organic solvents.

On the other hand, due to the diverse biological properties of barbituric acid derivatives (1), there is a widespread interest in their synthesis [3-7]. Compounds alkylated in the fifth position have demonstrated anticancer, HIV-1 and HIV-2 protease inhibitors [8], sedative-hypnotic $[9,10]$ and anticonvulsant [11] properties. Many of their representatives have clinical use as anti-inflammatory [12] and hypnotic drugs, such as veronal, phenobarbital, seconal, bucolone and sodium pentothal (Figure 1) [13-15]. A
() Chemistry Central 
<smiles>CCCCCCc1c(O)n(C2CCCCC2)c(=O)[nH]c1=O</smiles>

Bucolome

(Antiinflamatory drug)<smiles>[R]c1ccccc1Cc1c(OC)[nH]c(=O)[nH]c1=O</smiles>

HIV-1 and HIV-2 Protease Inhibitors<smiles>Cn1c(=O)[nH]c(N2CCC(Cc3ccccc3)CC2)c(Cc2ccccc2Br)c1=O</smiles>

HIV-1 and HIV-2

Protease Inhibitor

Figure 1 Bioactive compounds containing the barbituric acid framework.

number of compounds having these systems have been synthesized with diverse pharmacological activities [16,17].

Dimedone (5,5-dimethylcyclohexane-1,3-dione) 2 belongs to the cyclic 1,3-diketones - a very important class of organic compounds. A wide range of practical applications of dimedone include their uses as versatile precursors for synthesis of numerous hetero and spirocyclic compounds [18], xanthene derivatives with their industrial [19] and synthetic [20] applications, and also as reagent for various analytical determinations [21].

As a part of our work on one-pot multicomponent reactions (MCRs) for the synthesis of various heterocyclic compounds, we report here a highly efficient procedure for the preparation of dimedone-barbituric derivatives based on tandem Aldol-Michael reactions using aqueous diethylamine medium.

\section{Results and discussion}

In a typical experimental procedure, a mixture of barbituric acid 1a,b, dimedone $\mathbf{2}$ and aromatic aldehyde $\mathbf{3}$ in water was stirred in the presence of a stoichiometric amount of diethylamine (1.0 equiv.) to afford the 'Zwitterion adduct salts' of dimedone-barbituric acid derivative 4a in high yields (Scheme 1).

A possible mechanism for the tandem Aldol- Michael reaction is shown in Figure 2. In the first step of the reaction, olefin is produced by a Aldol condensation between aryl aldehyde $\mathbf{3}$ and $\mathbf{1} \mathbf{a}, \mathbf{b}$ promoted by DEA. Dimedone in the presence of DEA is then converted to its corresponding diethylammonium dimedonate that easily reacts with olefin to give product 4a-p [22-31].

In the absence of DEA, the reaction does not proceed efficiently and only a poor yield of products was obtained after $10 \mathrm{~h}$. The structures of products were confirmed by physical and spectroscopic (IR, MS, NMR) data, and by elemental analysis. The workup procedure is very simple and the products do not require further purification.

The X-ray zwitterion structure of $\mathbf{4 a}$ (Figure 3) was obtained using $\mathrm{X}$-ray structure determination from a single crystal grown from $\mathrm{CHCl}_{3} / \mathrm{Et}_{2} \mathrm{O}$ as solvents. The structure shows interesting characteristics (Table 1). We were unable to determine the location of the $\mathrm{C} 6$ and $\mathrm{C} 14$ hydrogens by ${ }^{1}$ HNMR analysis. This is because the hydrogen from C6 dimedone, rather than hydrogen from $\mathrm{C} 14$ of the barbituric acid moiety, is removed by the basicity of diethylamine. This was confirmed by the X-ray structure because one hydrogen is on the diethylamine and the other is involved in hydrogen bonding interactions between both barbituric acid and dimedone moiety. The hydrogen-bonding interactions are listed in Table 2. Figure 4 depicts the packing of the molecules in the crystal structure. The crystal structure is stabilized by $\mathrm{C}-\mathrm{H}$. . . O hydrogen bonds into a three-dimensional framework structure. It is noteworthy to mention that ${ }^{1} \mathrm{HNMR}$ have also shown a singlet signal at $\delta 15.28 \mathrm{ppm}$ which can be assigned to the $\mathrm{OH}$ group which makes a hydrogen bond.

With the optimal reaction conditions established, the generality of the Aldol-Michael reactions was next investigated by using a series of aryl aldehyde 3 (Table 3). Various aldehydes derivatives with either electron-withdrawing or electron-donating groups at the para-, meta-, or even sterically hindered ortho-position on the aromatic ring were tolerated and gave the corresponding condensed products 4a-p in excellent chemical yield up to 98\% (Scheme 2).

\section{Conclusions}

In summary, a mild, efficient, and expeditious method has been developed for the synthesis of zwitterion-condensed products 4a-p via a three component; one-pot cyclocondensation reaction of aromatic aldehyde, barbituric acid, and dimedone using aqueous diethylamine medium. The main advantage of the present methodology is a simple work-up procedure with milder reaction conditions. This method provides excellent yields of the products with high selectivity. Further studies on expanding the application of this method and the biological evaluation of these dimedone-barbituric derivatives are in progress.

\section{Experimental section}

General

All chemicals were purchased from Aldrich, Sigma-Aldrich, Fluka etc., and were used without further purification, 
<smiles>[R7]N1C(=O)CC(=O)N([R7])C1=O</smiles>

unless otherwise stated. All melting points were measured on a Gallenkamp melting point apparatus in open glass capillaries and are uncorrected. IR Spectra were measured as $\mathrm{KBr}$ pellets on a Nicolet 6700 FT-IR spectrophotometer. The NMR spectra were recorded on a Jeol-400 NMR spectrometer. ${ }^{1} \mathrm{H}$ NMR (400 MHz), and ${ }^{13} \mathrm{C}$ NMR $(100 \mathrm{MHz})$ were run in either deuterated dimethylsulphoxide (DMSO- $\left.d_{6}\right)$ or deuterated chloroform $\left(\mathrm{CDCl}_{3}\right)$. Chemical shifts $(\delta)$ are referred in terms of ppm and $J$-coupling constants are given in $H z$. Mass spectra were recorded on a Jeol of JMS-600H. Elemental analysis was carried out on an Elmer 2400 Elemental Analyzer; CHN mode.

\section{General procedure for aldol condensation Michael addition} for the synthesis of 4a-p (GP1)

A mixture of aldehyde 3 (1.5 mmol), dimedone 2 $(1.5 \mathrm{mmol})$, barbituric acid derivatives $\mathbf{1 a}, \mathbf{b}(1.5 \mathrm{mmol})$ and $\mathrm{Et}_{2} \mathrm{NH}(1.5 \mathrm{mmol}, 155 \mu \mathrm{L})$ in $1.5 \mathrm{~mL}$ of degassed $\mathrm{H}_{2} \mathrm{O}$ was stirred at room temperature for 1-2 hours until TLC showed complete disappearance of the reactants. The product precipitated and the mixture was filtered and washed with ether $(3 \times 20 \mathrm{~mL})$. The solid was recrystallized from a mixture of $\mathrm{CH}_{2} \mathrm{Cl}_{2} / \mathrm{Et}_{2} \mathrm{O}$ to afford pure product 4a-p.

\section{5-((2-Hydroxy-4,4-dimethyl-6-oxocyclohex-1-en-1-yl)(phenyl) methyl)-1,3-dimethyl-2,6-dioxo-1,2,3,6-tetrahydropyrimidin-4- olate (4a)}

4a was prepared from 1,3-dimethylbarbituric acid 1a, dimedone 2 and benzaldehyde according to the general procedure (GP1) yielding colorless crystalline material (671 mg, $1.47 \mathrm{mmol}, 98 \%)$. m.p: $159^{\circ} \mathrm{C}$; IR $\left(\mathrm{KBr}, \mathrm{cm}^{-1}\right)$ : 3150, 2959, 1667, 1617, 1585, 1422, 1256, 1227; ${ }^{1} \mathrm{H}$ NMR $\left(400 \mathrm{MHz}, \mathrm{CDCl}_{3}\right): \delta 15.28(\mathrm{~s}, 1 \mathrm{H}, \mathrm{OH}), 7.17-7.04(\mathrm{~m}, 5 \mathrm{H}$, $\mathrm{Ph}), 5.85\left(\mathrm{~s}, 1 \mathrm{H}\right.$, benzyl-H), $3.29\left(\mathrm{~s}, 12 \mathrm{H}, 4 \mathrm{CH}_{3}\right), 2.96(\mathrm{q}$, $\left.4 \mathrm{H}, J=7.3 \mathrm{~Hz}, \mathrm{CH}_{2} \mathrm{CH}_{3}\right), 2.42\left(\mathrm{~d}, 2 \mathrm{H}, J=5.1 \mathrm{~Hz}, \mathrm{CH}_{2}\right)$, $2.29\left(\mathrm{~m}, 2 \mathrm{H}, \mathrm{CH}_{2}\right), 1.24$ (t, $\left.6 \mathrm{H}, J=7.3 \mathrm{~Hz}, \mathrm{CH}_{2} \mathrm{CH}_{3}\right), 1.14$ (s, $\left.3 \mathrm{H}, \mathrm{CH}_{3}\right), 1.05\left(\mathrm{~s}, 3 \mathrm{H}, \mathrm{CH}_{3}\right) ;{ }^{13} \mathrm{C}$ NMR $(100 \mathrm{MHz}$, $\left.\mathrm{CDCl}_{3}\right): \delta=192.5,180.8,152.5,142.5,128.0,126.7,125.1$, 116.3, 90.9, 51.4, 45.9, 42.2, 33.0, 31.5, 29.6, 28.4, 27.6, 11.4; 


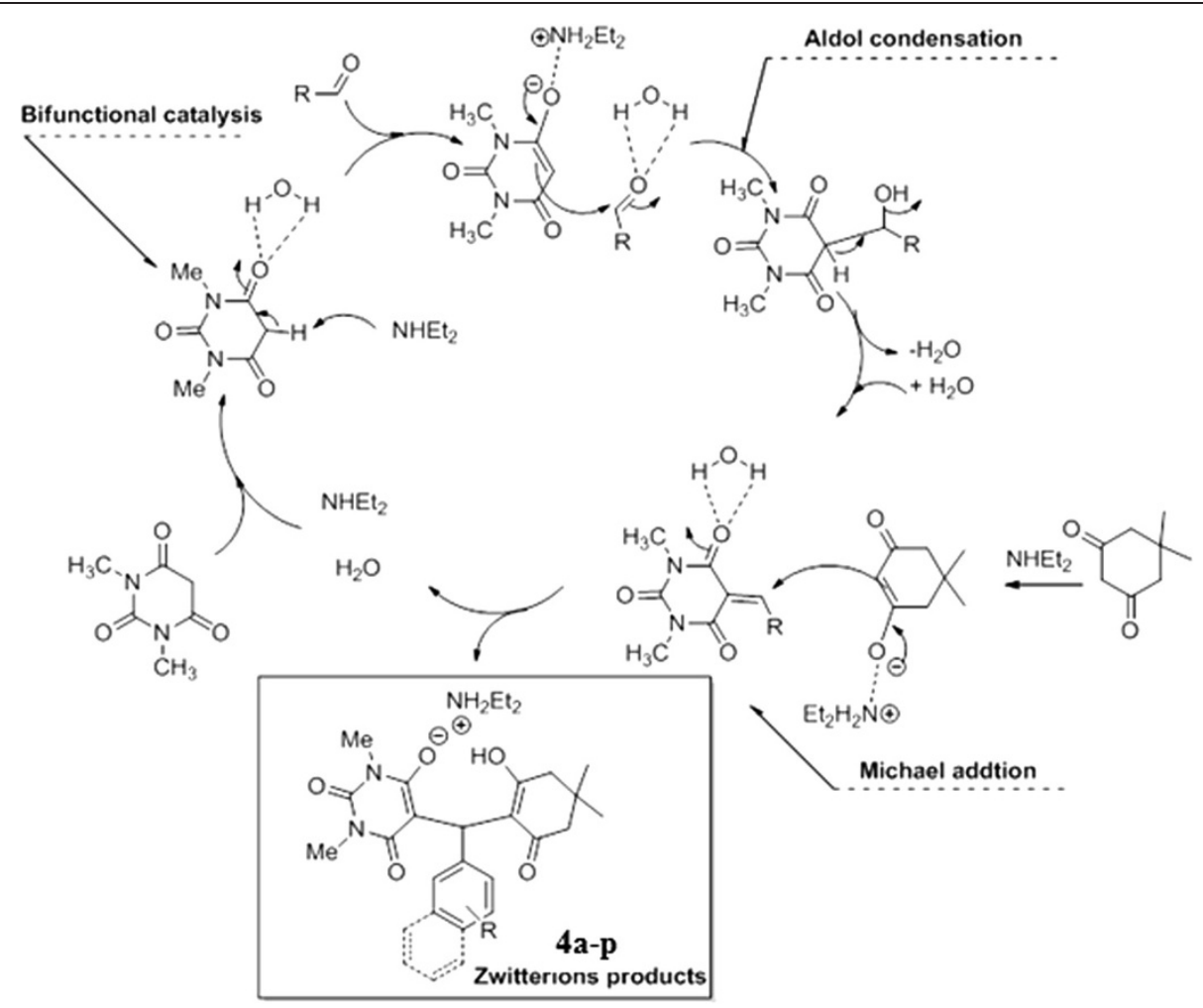

Figure 2 A possible mechanistic pathway.

LC/MS (ESI): $457[\mathrm{M}]^{+}$; Anal. for $\mathrm{C}_{25} \mathrm{H}_{35} \mathrm{~N}_{3} \mathrm{O}_{5}$; calcd: C, 65.62; H, 7.71; N, 9.18; Found: C, 65.61; H, 7.73; N, 9.20.

The structure of $\mathbf{4 a}$ was confirmed by X-ray crystal structure analysis. CCDC- 933624 contains the supplementary crystallographic data for this compound. This data can be obtained free of charge from the Cambridge Crystallographic Data Centre via www.ccdc.cam.ac.uk/data_request/cif. A colorless crystal suitable for X-ray analysis was obtained from recrystallization of the compound from $\mathrm{CHCl}_{3} / \mathrm{Et}_{2} \mathrm{O}$ at room temperature after 2 days.

\section{5-((2-Hydroxy-4,4-dimethyl-6-oxocyclohex-1-en-1-yl)(p-tolyl) methyl)-1,3-dimethyl-2,6-dioxo-1,2,3,6-tetrahydropyrimidin-4- olate (4b)}

4b was prepared from 1,3-dimethylbarbituric acid 1a, dimedone $\mathbf{2}$ and p-tolualdehyde according to the general procedure (GP1) yielding an oily material $(685 \mathrm{mg}$, $1.45 \mathrm{mmol}, 97 \%)$. IR (KBr, $\left.\mathrm{cm}^{-1}\right): 3150,2954,2867,1675$, 1580, 1508, 1447, 1380, 1256, 1145; ${ }^{1} \mathrm{H}$ NMR (400 MHz, $\left.\mathrm{CDCl}_{3}\right): \delta 15.25$ (s, 1H, OH), 7.00-6.93 (m, 4H, Ph), 5.84 (s, $1 \mathrm{H}$, benzyl-H), $3.28\left(\mathrm{~s}, 12 \mathrm{H}, 4 \mathrm{CH}_{3}\right), 2.90$ (q, 4H, J= 7.3 $\left.\mathrm{Hz}, \mathrm{CH}_{2} \mathrm{CH}_{3}\right), 2.30$ (d, $\left.4 \mathrm{H}, J=5.1 \mathrm{~Hz}, \mathrm{CH}_{2}\right), 2.22(\mathrm{~s}, 3 \mathrm{H}$, $\left.\mathrm{CH}_{3}\right), 1.20\left(\mathrm{t}, 6 \mathrm{H}, J=7.3 \mathrm{~Hz}, \mathrm{CH}_{2} \mathrm{CH}_{3}\right), 1.16\left(\mathrm{~s}, 3 \mathrm{H}, \mathrm{CH}_{3}\right)$, $1.04\left(\mathrm{~s}, 3 \mathrm{H}, \mathrm{CH}_{3}\right) ;{ }^{13} \mathrm{C} \mathrm{NMR}\left(100 \mathrm{MHz}, \mathrm{CDCl}_{3}\right): \delta=196.5$, $180.1,152.8,140.5,134.2,129.8,128.7,126.8,126.7,115.6$, 91.0, 51.4, 45.9, 42.5, 32.6, 31.5, 29.6, 28.4, 27.6, 20.9, 11.9; LC/MS (ESI): $471[\mathrm{M}]^{+}$; Anal. for $\mathrm{C}_{26} \mathrm{H}_{37} \mathrm{~N}_{3} \mathrm{O}_{5}$; calcd: C, 66.22; H, 7.91; N, 8.91; Found: C, 66.24; H, $7.92 ; \mathrm{N}, 8.87$.

5-((4-Chlorophenyl)(2-hydroxy-4,4-dimethyl-6-oxocyclohex1-en-1-yl)methyl)-1,3-dimethyl-2,6-dioxo-1,2,3,6tetrahydropyrimidin-4-olate (4c)

4c was prepared from 1,3-dimethylbarbituric acid 1a, dimedone $\mathbf{2}$ and $p$-chlorobenzaldehyde $\mathbf{3}$ according to the general procedure (GP1) yielding an oily material (715 mg, $1.45 \mathrm{mmol}, 97 \%)$. IR (KBr, $\left.\mathrm{cm}^{-1}\right)$ : 3151, 2955, 2868, 2497, 1675, 1580, 1481, 1444, 1379, 1258, 1206; ${ }^{1} \mathrm{H}$ NMR (400 MHz, $\left.\mathrm{CDCl}_{3}\right): \delta 15.02(\mathrm{~s}, 1 \mathrm{H}, \mathrm{OH}), 7.12-6.95$ (m, $4 \mathrm{H}, \mathrm{Ph}), 5.87$ (s, 1H, benzyl-H), $3.30\left(\mathrm{~s}, 12 \mathrm{H}, 4 \mathrm{CH}_{3}\right)$, $2.90\left(\mathrm{q}, 4 \mathrm{H}, J=7.3 \mathrm{~Hz}, \mathrm{CH}_{2} \mathrm{CH}_{3}\right), 2.38\left(\mathrm{~s}, 4 \mathrm{H}, \mathrm{CH}_{2}\right)$, $1.20\left(\mathrm{t}, 6 \mathrm{H}, J=7.3 \mathrm{~Hz}, \mathrm{CH}_{2} \mathrm{CH}_{3}\right), 1.16\left(\mathrm{~s}, 3 \mathrm{H}, \mathrm{CH}_{3}\right), 1.04$ $\left(\mathrm{s}, 3 \mathrm{H}, \mathrm{CH}_{3}\right) ;{ }^{13} \mathrm{C} \mathrm{NMR}\left(100 \mathrm{MHz}, \mathrm{CDCl}_{3}\right): \delta=198.1$, 181.0, 152.5, 141.5, 130.6, 128.3, 128.2, 128.0, 127.9, 115.2, 90.7, 65.9, 49.8, 42.3, 32.4, 31.5, 31.2, 29.6, 28.4, 27.6, 15.3, 11.4; LC/MS (ESI): $492[\mathrm{M}]^{+}$; Anal. for $\mathrm{C}_{25} \mathrm{H}_{34 \mathrm{Cl}} \mathrm{N}_{3} \mathrm{O}_{5}$; calcd: C, 61.03; H, 6.97; Cl, 7.21; N, 8.54; Found: C, 61.06; H, 7.00; Cl, 7.18; N, 8.57.

\section{5-((4-Bromophenyl)(2-hydroxy-4,4-dimethyl-6-oxocyclohex-}

1-en-1-yl)methyl)-1,3-dimethyl-2,6-dioxo-1,2,3,6-

tetrahydropyrimidin-4-olate (4d)

4d was prepared from 1,3-dimethylbarbituric acid 1a, dimedone $\mathbf{2}$ and $p$-bromobenzaldehyde $\mathbf{3}$ according to the 


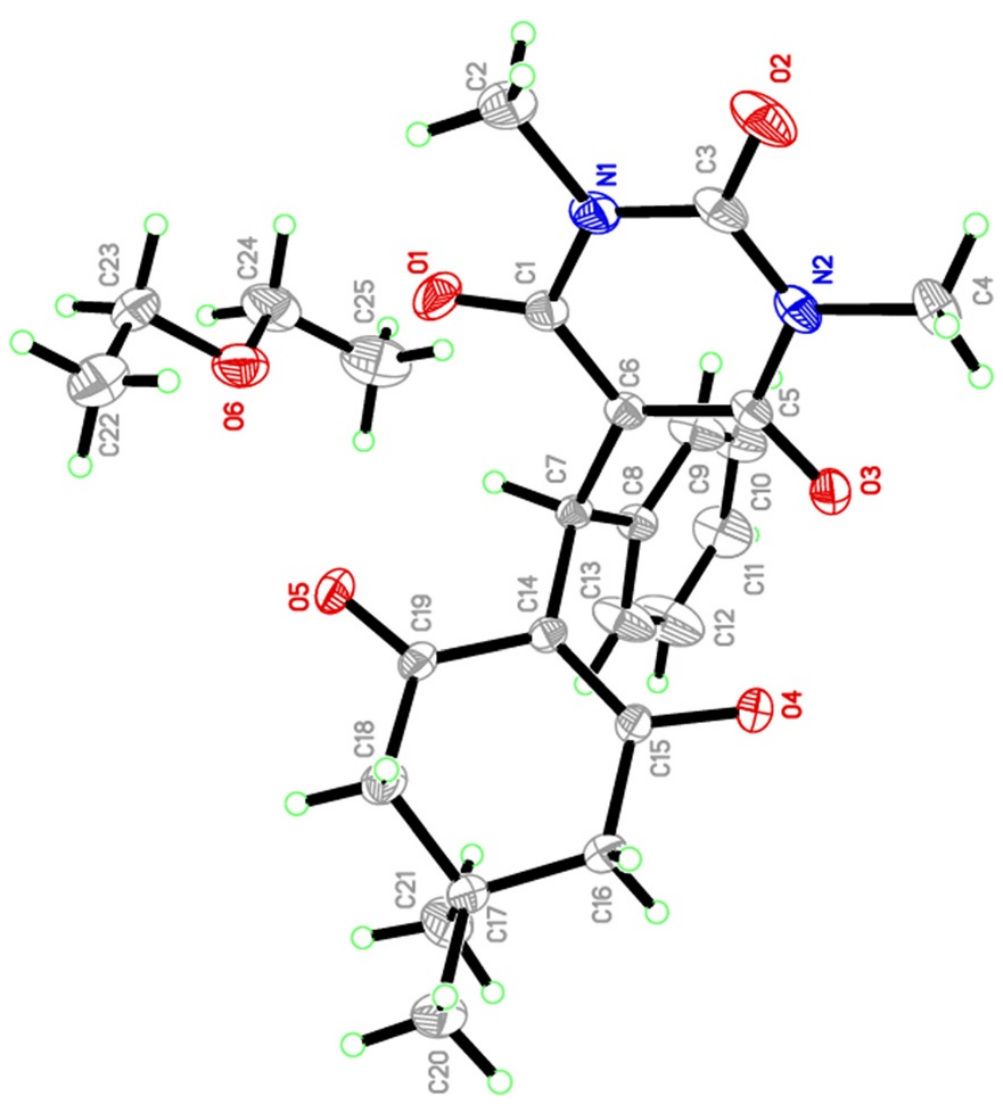

Figure 3 ORTEP representation of the structure of $4 a$.

general procedure (GP1) yielding an oily material (761 mg, $1.42 \mathrm{mmol}, 95 \%)$. IR (KBr, $\left.\mathrm{cm}^{-1}\right)$ : 3155, 2955, 2867, 2500, 1674, 1579, 1430, 1376, 1204; ${ }^{1} \mathrm{H}$ NMR $(400 \mathrm{MHz}$, $\left.\mathrm{CDCl}_{3}\right): \delta 15.20(\mathrm{~s}, 1 \mathrm{H}, \mathrm{OH}), 7.34(\mathrm{~d}, 2 \mathrm{H}, J=8.0 \mathrm{~Hz}$, $\mathrm{Ph}), 6.98(\mathrm{~d}, 2 \mathrm{H}, J=8.0 \mathrm{~Hz}, \mathrm{Ph}), 5.79$ (s, $1 \mathrm{H}$, benzyl-H), 3.27 (s, 12H, 4CH $\mathrm{CH}_{3}, 2.99$ (q, $4 \mathrm{H}, J=7.3 \mathrm{~Hz}, \mathrm{CH}_{2} \mathrm{CH}_{3}$ ), 2.40 $\left(\mathrm{d}, 2 \mathrm{H}, J=5.1 \mathrm{~Hz}, \mathrm{CH}_{2}\right), 2.28\left(\mathrm{~m}, 2 \mathrm{H}, \mathrm{CH}_{2}\right), 1.29(\mathrm{t}, 6 \mathrm{H}, J=$ $\left.7.3 \mathrm{~Hz}, \mathrm{CH}_{2} \mathrm{CH}_{3}\right), 1.18\left(\mathrm{~s}, 3 \mathrm{H}, \mathrm{CH}_{3}\right), 1.04\left(\mathrm{~s}, 3 \mathrm{H}, \mathrm{CH}_{3}\right)$; ${ }^{13} \mathrm{C}$ NMR $\left(100 \mathrm{MHz}, \mathrm{CDCl}_{3}\right): \delta=199.1,191.2,164.8$, $152.4,142.8,132.5,131.0,129.9,128.7,128.6,118.9$, $115.9,90.6,51.2,45.8,42.3,32.7,31.5,29.5,28.5$, 28.3, 27.6, 11.4; LC/MS (ESI): $536[\mathrm{M}]^{+}$; Anal. for $\mathrm{C}_{25} \mathrm{H}_{34} \mathrm{BrN}_{3} \mathrm{O}_{5}$; calcd: C, 55.97; $\mathrm{H}, 6.39 ; \mathrm{Br}, 14.89 ; \mathrm{N}$, 7.83; Found: C, 56.00; H, 6.40; Br, 14.86; N, 7.82.

\section{5-((3-Bromophenyl)(2-hydroxy-4,4-dimethyl-6-oxocyclohex- 1-en-1-yl)methyl)-1,3-dimethyl-2,6-dioxo-1,2,3,6- tetrahydropyrimidin-4-olate (4e)}

4e was prepared from 1,3-dimethylbarbituric acid 1a, dimedone $\mathbf{2}$ and $m$-bromobenzaldehyde $\mathbf{3}$ according to the general procedure (GP1) yielding an oily material (745 mg, $1.39 \mathrm{mmol}, 93 \%)$. IR ( $\left.\mathrm{KBr}, \mathrm{cm}^{-1}\right)$ : 3050, 2955, 2868, 2500, 1675, 1581, 1444, 1378, 1255, 1205; ${ }^{1} \mathrm{H}$ NMR (400 MHz, $\left.\mathrm{CDCl}_{3}\right): \delta 15.63(\mathrm{~s}, 1 \mathrm{H}, \mathrm{OH}), 7.22(\mathrm{~d}$, $1 \mathrm{H}, J=7.3 \mathrm{~Hz}, \mathrm{Ph}), 7.19$ (s, 1H, Ph), 7.07 (d, 1H, $J=7.3 \mathrm{~Hz}$,
$\mathrm{Ph}), 7.05$ (d, 1H, J=7.3 Hz, Ph), $5.84(\mathrm{~s}, 1 \mathrm{H}$, benzyl-H), $3.34\left(\mathrm{~s}, 6 \mathrm{H}, 2 \mathrm{CH}_{3}\right), 3.32\left(\mathrm{~s}, 6 \mathrm{H}, 2 \mathrm{CH}_{3}\right), 2.98(\mathrm{q}, 4 \mathrm{H}, J=$ $\left.7.3 \mathrm{~Hz}, \mathrm{CH}_{2} \mathrm{CH}_{3}\right), 2.31\left(\mathrm{~d}, 4 \mathrm{H}, J=5.1 \mathrm{~Hz}, \mathrm{CH}_{2}\right), 1.24(\mathrm{t}, 6 \mathrm{H}$, $\left.J=7.3 \mathrm{~Hz}, \mathrm{CH}_{2} \mathrm{CH}_{3}\right), 1.12\left(\mathrm{~s}, 3 \mathrm{H}, \mathrm{CH}_{3}\right), 1.03(\mathrm{~s}, 3 \mathrm{H}$, $\left.\mathrm{CH}_{3}\right) ;{ }^{13} \mathrm{C}$ NMR $\left(100 \mathrm{MHz}, \mathrm{CDCl}_{3}\right): \delta=190.8,186.4$, 165.2, 164.4, 151.7, 144.7, 129.7,129.6, 128.7, 125.3, 91.5, 42.1, 34.4, 28.9, 28.7, 11.5; LC/MS (ESI): $536\left[\mathrm{M}^{+}\right.$; Anal. for $\mathrm{C}_{25} \mathrm{H}_{34} \mathrm{BrN}_{3} \mathrm{O}_{5}$; calcd: C, 55.97; $\mathrm{H}, 6.39 ; \mathrm{Br}, 14.89 ; \mathrm{N}$, 7.83; Found: C, 56.01; H, 6.41; Br, 14.86; N, 7.84.

\section{5-((2-Hydroxy-4,4-dimethyl-6-oxocyclohex-1-en-1-yl)} (4-methoxyphenyl)methyl)-1,3-dimethyl-2,6-dioxo-1,2,3, 6-tetrahydropyrimidin-4-olate (4f)

4f was prepared from 1,3-dimethylbarbituric acid 1a, dimedone $\mathbf{2}$ and anisaldehyde $\mathbf{3}$ according to the general procedure (GP1) yielding an oily material (672 mg, $1.38 \mathrm{mmol}, 92 \%)$. IR (KBr, $\left.\mathrm{cm}^{-1}\right)$ : 3047, 2953, 2866, 2499, 1679, 1577, 1510, 1427, 1373, 1255, 1214; ${ }^{1} \mathrm{H}$ NMR $\left(400 \mathrm{MHz} \mathrm{CDCl}_{3}\right): \delta 15.26(\mathrm{~s}, 1 \mathrm{H}, \mathrm{OH}), 6.98(\mathrm{~d}, 2 \mathrm{H}, J=$ $8.0 \mathrm{~Hz}, \mathrm{Ph}), 6.72(\mathrm{~d}, 2 \mathrm{H}, J=8.0 \mathrm{~Hz}, \mathrm{Ph}), 5.69(\mathrm{~s}, 1 \mathrm{H}$, benzyl-H), $3.71\left(\mathrm{~s}, 3 \mathrm{H}, \mathrm{CH}_{3}\right), 3.29\left(\mathrm{~s}, 12 \mathrm{H}, 4 \mathrm{CH}_{3}\right), 2.87$ (q, $\left.4 \mathrm{H}, J=7.3 \mathrm{~Hz}, \mathrm{CH}_{2} \mathrm{CH}_{3}\right), 2.31\left(\mathrm{~d}, 4 \mathrm{H}, J=5.1 \mathrm{~Hz}, \mathrm{CH}_{2}\right.$ ), $1.19\left(\mathrm{t}, 6 \mathrm{H}, J=7.3 \mathrm{~Hz}, \mathrm{CH}_{2} \mathrm{CH}_{3}\right), 1.12\left(\mathrm{~s}, 3 \mathrm{H}, \mathrm{CH}_{3}\right), 1.03$ $\left(\mathrm{s}, 3 \mathrm{H}, \mathrm{CH}_{3}\right) ;{ }^{13} \mathrm{C} \mathrm{NMR}\left(100 \mathrm{MHz}, \mathrm{CDCl}_{3}\right): \delta=195.1$, 187.2 , 157.1, 134.5, 133.9, 127.8, 127.6, 115.6, 113.4, 


\begin{tabular}{|c|c|}
\hline Empirical formula & $\mathrm{C}_{25} \mathrm{H}_{32} \mathrm{~N}_{2} \mathrm{O}_{6}$ \\
\hline Formula weight & 456.53 \\
\hline Temperature (K) & 293 \\
\hline Crystal system & Orthorhombic \\
\hline Space group & Pbca \\
\hline Cu Ka radiation, $\lambda$ & $1.54178 \AA$ \\
\hline$a=$ & $14.6669(5) \AA$ \\
\hline$b=$ & $18.3084(6) \AA$ \\
\hline$c=$ & $19.0294(6) \AA$ \\
\hline$a=$ & $90^{\circ}$ \\
\hline$\beta=$ & $90^{\circ}$ \\
\hline$\gamma=$ & $90^{\circ}$ \\
\hline$V=$ & $5109.9(3) \AA^{3}$ \\
\hline$Z=$ & 8 \\
\hline Theta range for data collection & $3.0-69.2^{\circ}$ \\
\hline$\mu=$ & $0.70 \mathrm{~mm}^{-1}$ \\
\hline Density clac. $\left(\mathrm{g} / \mathrm{cm}^{3}\right)$ & 1.187 \\
\hline Crystal shape and colour & Plate, colourless \\
\hline Crystal size & $0.89 \times 0.78 \times 0.22 \mathrm{~mm}$ \\
\hline$h / k / l$ & $-17,17 /-22,22 /-22,23$ \\
\hline Measured reflections & 32924 \\
\hline Independent reflections & $4796\left(R_{\text {int }}=0.088\right)$ \\
\hline Reflections with $1>2 \sigma()$ & 3997 \\
\hline Goodness-of-fit on $F^{2}$ & 1.04 \\
\hline$R\left[F^{2}>2 \sigma\left(F^{2}\right)\right]=$ & 0.067 \\
\hline$w R\left(F^{2}\right)=$ & 0.195 \\
\hline$\Delta \rho_{\max }=$ & 0.47 e $\AA^{-3}$ \\
\hline$\Delta \rho_{\min }=$ & -0.40 e $\AA^{-3}$ \\
\hline
\end{tabular}

55.2, 42.6, 31.5, 31.1, 27.9, 12.2; LC/MS (ESI): 487 [M] ${ }^{+}$; Anal. for $\mathrm{C}_{26} \mathrm{H}_{37} \mathrm{~N}_{3} \mathrm{O}_{6}$; calcd: C, 64.05; H, 7.65; N, 8.62; Found: C, 64.11; H, 7.64; N, 8.59.

\section{5-((2-Hydroxy-4,4-dimethyl-6-oxocyclohex-1-en-1-yl) (4-nitrophenyl)methyl)-1,3-dimethyl-2,6-dioxo-1,2,3, 6-tetrahydropyrimidin-4-olate (4 g)}

4b was prepared from 1,3-dimethylbarbituric acid 1a, dimedone $\mathbf{2}$ and $p$-nitrobenzaldehyde $\mathbf{3}$ according to the

Table 2 Hydrogen-bond geometry $\left(\AA{ }^{\circ}{ }^{\circ}\right)$

\begin{tabular}{|c|c|c|c|c|}
\hline$D-\mathrm{H} \cdots A$ & $D-\mathrm{H}$ & $\mathrm{H} \cdots A$ & $D \cdots A$ & $D-\mathrm{H} \cdots A$ \\
\hline $\mathrm{C} 2-\mathrm{H} 2 \mathrm{~B} \cdots \mathrm{O} 1$ & 0.9600 & 2.2600 & $2.655(3)$ & 104.00 \\
\hline $\mathrm{C} 4-\mathrm{H} 4 \mathrm{~B} \cdots \mathrm{O} 3$ & 0.9600 & 2.2300 & $2.682(3)$ & 108.00 \\
\hline $\mathrm{C} 7-\mathrm{H} 7 \mathrm{~A} \cdots \mathrm{O} 1$ & 0.9800 & 2.3700 & $2.894(2)$ & 113.00 \\
\hline $\mathrm{C} 7-\mathrm{H} 7 \mathrm{~A} \cdots \mathrm{O} 5$ & 0.9800 & 2.2800 & $2.821(2)$ & 114.00 \\
\hline $\mathrm{C} 22-\mathrm{H} 22 \mathrm{~A} \cdots \mathrm{O}^{\mathrm{i}}$ & 0.9600 & 2.5400 & $3.376(3)$ & 146.00 \\
\hline
\end{tabular}

Symmetry code: (i) $x,-y+3 / 2, z+1 / 2$. general procedure (GP1) yielding a beige material $(700 \mathrm{mg}$, 1.39 mmol, 93\%). m.p: $148^{\circ} \mathrm{C}$; IR $\left(\mathrm{KBr}, \mathrm{cm}^{-1}\right)$ : 3050, 2950, $2865,2500,1669,1580,1510,1427,1373,1255,1214,{ }^{1} \mathrm{H}$ NMR (400 MHz, $\left.\mathrm{CDCl}_{3}\right): \delta 15.26(\mathrm{~s}, 1 \mathrm{H}, \mathrm{OH}), 6.99$ (d, $2 \mathrm{H}, J=8.0 \mathrm{~Hz}, \mathrm{Ph}), 6.72$ (d, $2 \mathrm{H}, J=8.8 \mathrm{~Hz}, \mathrm{Ph}), 5.69$ (s, $1 \mathrm{H}$, benzyl-H), $3.71\left(\mathrm{~s}, 12 \mathrm{H}, 4 \mathrm{CH}_{3}\right), 2.85(\mathrm{q}, 4 \mathrm{H}, J=7.3 \mathrm{~Hz}$, $\left.\mathrm{CH}_{2} \mathrm{CH}_{3}\right), 2.31\left(\mathrm{~d}, 4 \mathrm{H}, J=14.7 \mathrm{~Hz}, \mathrm{CH}_{2}\right), 1.19(\mathrm{t}, 6 \mathrm{H}, J=7.3$ $\left.\mathrm{Hz}, \mathrm{CH}_{2} \mathrm{CH}_{3}\right), 1.12\left(\mathrm{~s}, 3 \mathrm{H}, \mathrm{CH}_{3}\right), 1.03\left(\mathrm{~s}, 3 \mathrm{H}, \mathrm{CH}_{3}\right) ;{ }^{13} \mathrm{C}$ NMR (100 MHz, $\left.\mathrm{CDCl}_{3}\right): \delta=161.6,153.2,145.5,141.6$, 129.1, 128.2, 127.8, 125.8, 88.5, 49.1, 41.9, 27.5, 11.5; LC/MS (ESI): $502[\mathrm{M}]^{+}$; Anal. for $\mathrm{C}_{25} \mathrm{H}_{34} \mathrm{~N}_{4} \mathrm{O}_{7}$; calcd: C, 59.75; $\mathrm{H}$, 6.82; N, 11.15; Found: C, 59.73; H, 6.81; N, 11.17.

5-((2,4-Dichlorophenyl)(2-hydroxy-4,4-dimethyl-6-oxocyclohex1-en-1-yl)methyl)-1,3-dimethyl-2,6-dioxo-1,2,3, 6-tetrahydropyrimidin-4-olate (4 h)

$4 \mathbf{h}$ was prepared from 1,3-dimethylbarbituric acid 1a, dimedone $\mathbf{2}$ and 2,4-dichlorobenzaldehyde 3 according to the general procedure (GP1) yielding a beige solid material (710 mg, $1.35 \mathrm{mmol}, 90 \%)$. m.p: $164^{\circ} \mathrm{C}$; IR $\left(\mathrm{KBr}, \mathrm{cm}^{-1}\right)$ : 3059, 2995, 2867, 2114, 1741, 1658, 1591, 1463, 1429, 1370, 1341, 1256, $1201^{1} \mathrm{H}-\mathrm{NMR}\left(400 \mathrm{MHz}, \mathrm{CDCl}_{3}\right): \delta 14.80$ (s, 1H, OH), 7.29 (d, 1H, J=8.0 Hz, Ph), $7.19(\mathrm{~s}, 1 \mathrm{H}, \mathrm{Ph})$, $7.12(\mathrm{~d}, 2 \mathrm{H}, J=8.0 \mathrm{~Hz}, \mathrm{Ph}), 5.76(\mathrm{~s}, 1 \mathrm{H}$, benzyl-H), 3.28 (s, $\left.12 \mathrm{H}, 4 \mathrm{CH}_{3}\right), 3.07$ (q, $\left.4 \mathrm{H}, J=7.3 \mathrm{~Hz}, \mathrm{CH}_{2} \mathrm{CH}_{3}\right), 2.37$ $\left(\mathrm{s}, 2 \mathrm{H}, \mathrm{CH}_{2}\right), 2.27\left(\mathrm{~d}, 2 \mathrm{H}, J=5.1 \mathrm{~Hz}, \mathrm{CH}_{2}\right), 1.34(\mathrm{t}, 6 \mathrm{H}, J=$ $\left.7.3 \mathrm{~Hz}, \mathrm{CH}_{2} \mathrm{CH}_{3}\right), 1.04\left(\mathrm{~s}, 3 \mathrm{H}, \mathrm{CH}_{3}\right), 1.01\left(\mathrm{~s}, 3 \mathrm{H}, \mathrm{CH}_{3}\right) ;{ }^{13} \mathrm{C}$ NMR $\left(100 \mathrm{MHz}, \mathrm{CDCl}_{3}\right): \delta=199.1,165.4,164.4,152.5$, $139.8,133.6,131.7,131.2,129.3,126.4,115.7,89.8,51.2$, 45.7, 41.9, 32.4, 31.2, 28.3, 28.2, 11.3; LC/MS (ESI): 526 $[\mathrm{M}]^{+}$; Anal. for $\mathrm{C}_{25} \mathrm{H}_{33} \mathrm{Cl}_{2} \mathrm{~N}_{3} \mathrm{O}_{5}$; calcd: $\mathrm{C}$, 57.04; $\mathrm{H}, 6.32$; Cl, 13.47; N, 7.98; Found: C, 57.09; H, 6.31; Cl, 13.44; N, 8.01 .

5-((2,6-Dichlorophenyl)(2-hydroxy-4,4-dimethyl-6-oxocyclohex1-en-1-yl)methyl)-1,3-dimethyl-2,6-dioxo-1,2,3,

6-tetrahydropyrimidin-4-olate (4i)

4i was prepared from 1,3-dimethylbarbituric acid 1a, dimedone 2 and 2,6-dichlorobenzaldehyde 3 according to the general procedure (GP1) yielding an oily material (702 mg, $1.33 \mathrm{mmol}, 89 \%)$. IR $\left(\mathrm{KBr}, \mathrm{cm}^{-1}\right): 3048,2955$, 2869, 2728, 2494, 1676, 1575, 1428, 1372, 1238, 1196; ${ }^{1} \mathrm{H}$ NMR $\left(400 \mathrm{MHz}, \mathrm{CDCl}_{3}\right): \delta 14.80(\mathrm{~s}, 1 \mathrm{H}, \mathrm{OH}), 7.36(\mathrm{~d}, 2 \mathrm{H}$, $J=8.0 \mathrm{~Hz}, \mathrm{Ph}), 7.29$ (t, $1 \mathrm{H}, J=8.0 \mathrm{~Hz}, \mathrm{Ph}), 7.12(\mathrm{~d}, 2 \mathrm{H}, J=$ $8.0 \mathrm{~Hz}, \mathrm{Ph}), 5.98(\mathrm{~s}, 1 \mathrm{H}$, benzyl- $\mathrm{H}), 3.26\left(\mathrm{~s}, 12 \mathrm{H}, 4 \mathrm{CH}_{3}\right), 2.92$ (q, $\left.4 \mathrm{H}, J=7.3 \mathrm{~Hz}, \mathrm{CH}_{2} \mathrm{CH}_{3}\right), 2.37\left(\mathrm{~s}, 2 \mathrm{H}, \mathrm{CH}_{2}\right), 2.27(\mathrm{~d}, 2 \mathrm{H}$, $\left.J=5.1 \mathrm{~Hz}, \mathrm{CH}_{2}\right), 1.24\left(\mathrm{t}, 6 \mathrm{H}, J=7.3 \mathrm{~Hz}, \mathrm{CH}_{2} \mathrm{CH}_{3}\right), 1.094$ $\left(\mathrm{s}, 3 \mathrm{H}, \mathrm{CH}_{3}\right), 1.04\left(\mathrm{~s}, 3 \mathrm{H}, \mathrm{CH}_{3}\right) ;{ }^{13} \mathrm{C}$ NMR $(100 \mathrm{MHz}$, $\left.\mathrm{CDCl}_{3}\right): \delta=192.8,188.9,165.3,164.3,152.5,149.7,137.4$, $131.5,129.8,126.5,124.2,115.5,114.7,89.9,53.5,41.4$, 31.9, 28.7, 28.2, 11.4 ; LC/MS (ESI): $526\left[\mathrm{MM}^{+}\right.$; Anal. for $\mathrm{C}_{25} \mathrm{H}_{33} \mathrm{Cl}_{2} \mathrm{~N}_{3} \mathrm{O}_{5}$; calcd: C, 57.04; $\mathrm{H}, 6.32 ; \mathrm{Cl}, 13.47 ; \mathrm{N}$, 7.98; Found: C, $57.08 ; \mathrm{H}, 6.30 ; \mathrm{Cl}, 13.45 ; \mathrm{N}, 8.00$. 


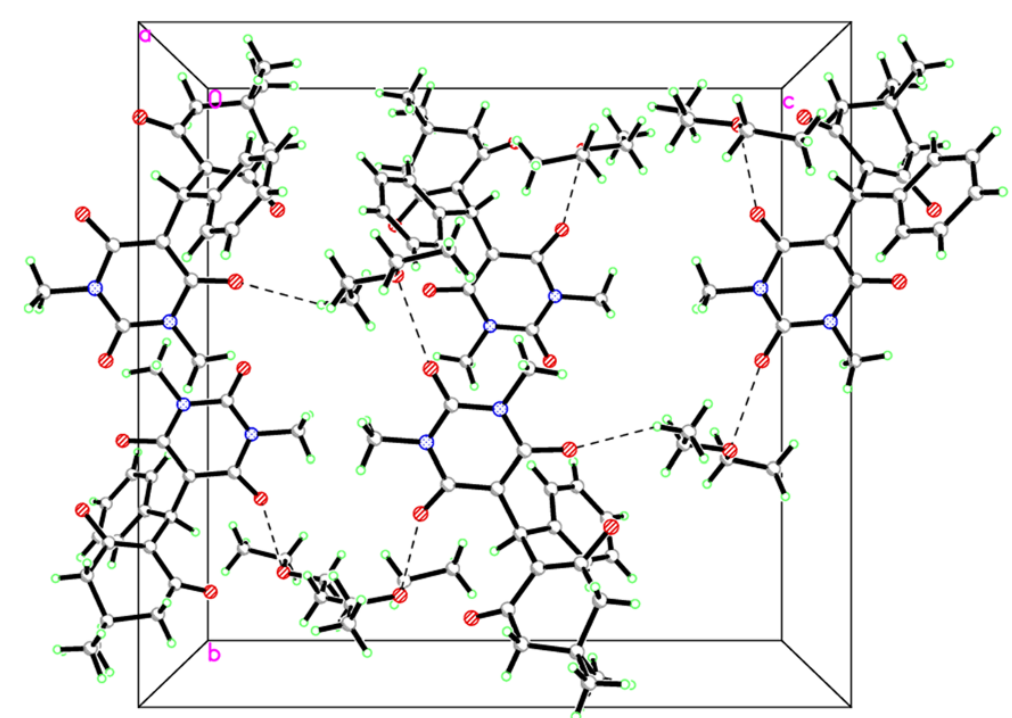

Figure 4 Crystal packing showing intermolecular $\mathrm{C}-\mathrm{H} \cdot \mathrm{O}$ hydrogen bonds as dashed lines. $4 \mathrm{a}$.

5-((2-Hydroxy-4,4-dimethyl-6-oxocyclohex-1-en-1-yl) (naphthalen-2-yl)methyl)-1,3-dimethyl-2,6-dioxo-1,2,3, 6-tetrahydropyrimidin-4-olate (4j)

$\mathbf{4 j}$ was prepared from 1,3-dimethylbarbituric acid 1a, dimedone $\mathbf{2}$ and 2-naphthaldehyde $\mathbf{3}$ according to the general procedure (GP1) yielding a white solid material $(715 \mathrm{mg}$, $1.41 \mathrm{mmol}, 94 \%)$ m.p: $170{ }^{\circ} \mathrm{C}$; IR $\left(\mathrm{KBr}, \mathrm{cm}^{-1}\right): 2994,2948$,

Table 3 Tandem Aldol-Michael reactions of barbituric acid $1 \mathrm{a}, \mathrm{b}$ and dimedone $\mathbf{2}$ with aldehydes 3 in aqueous diethylamine medium ${ }^{a}$

\begin{tabular}{|c|c|c|c|c|}
\hline$\#$ & 3 & $\mathrm{R}_{1}$ & $\mathbf{R}_{\mathbf{2}}$ & ${\text { yield }(\%)^{b}}^{b}$ \\
\hline 1 & $4 a$ & $\mathrm{CH}_{3}$ & $\mathrm{Ph}$ & 98 \\
\hline 2 & $4 b$ & $\mathrm{CH}_{3}$ & $\mathrm{p}-\mathrm{CH}_{3} \mathrm{Ph}$ & 97 \\
\hline 3 & $4 c$ & $\mathrm{CH}_{3}$ & $p-C I P h$ & 97 \\
\hline 4 & $4 d$ & $\mathrm{CH}_{3}$ & p-BrPh & 95 \\
\hline 5 & $4 e$ & $\mathrm{CH}_{3}$ & $m-\mathrm{BrPh}$ & 93 \\
\hline 6 & $4 f$ & $\mathrm{CH}_{3}$ & $p-\mathrm{CH}_{3} \mathrm{OPh}$ & 92 \\
\hline 7 & $4 \mathrm{~g}$ & $\mathrm{CH}_{3}$ & $\mathrm{O}-\mathrm{NO}_{2} \mathrm{Ph}$ & 93 \\
\hline 8 & $4 \mathrm{~h}$ & $\mathrm{CH}_{3}$ & $2,4-\mathrm{Cl}_{2} \mathrm{Ph}$ & 90 \\
\hline 9 & $4 i$ & $\mathrm{CH}_{3}$ & $2,6-\mathrm{Cl}_{2} \mathrm{Ph}$ & 89 \\
\hline 10 & $4 j$ & $\mathrm{CH}_{3}$ & 2-Naphthaldehyde & 94 \\
\hline 11 & $4 \mathrm{k}$ & $\mathrm{CH}_{3}$ & p-HO-Ph & 91 \\
\hline 12 & 41 & $\mathrm{H}$ & $\mathrm{Ph}$ & 93 \\
\hline 13 & $4 \mathrm{~m}$ & $\mathrm{H}$ & $p-\mathrm{CH}_{3} \mathrm{Ph}$ & 91 \\
\hline 14 & $4 n$ & $\mathrm{H}$ & $p-C l P h$ & 90 \\
\hline 15 & 40 & $\mathrm{H}$ & $p$-BrPh & 89 \\
\hline 16 & $4 p$ & $\mathrm{H}$ & 2-Naphthaldehyde & 90 \\
\hline
\end{tabular}

${ }^{\mathrm{a}}$ All reactions were carried out with barbituric acid derivatives $\mathbf{1} \mathbf{a}, \mathbf{b}(1.5 \mathrm{mmol})$, dimedone $2(1.5 \mathrm{mmol})$ aldehydes $\mathbf{3}(1.5 \mathrm{mmol})$ and diethylamine $(1.5 \mathrm{mmol})$ in water $(1.5 \mathrm{~mL})$ for the specified time. ${ }^{\mathrm{b}}$ Yield of isolated product $4 \mathbf{a - p}$.
2866, 2506, 1742, 1651, 1603, 1570, 1526, 1473, 1431, 1362, $1245 ;{ }^{1} \mathrm{H}$ NMR (400 MHz, $\left.\mathrm{CDCl}_{3}\right): \delta 14.26(\mathrm{~s}, 1 \mathrm{H}$, $\mathrm{OH}), 7.46-7.22(\mathrm{~m}, 7 \mathrm{H}$, naphthyl), 6.20 (s, $1 \mathrm{H}$, benzyl-H), $3.26\left(\mathrm{~s}, 6 \mathrm{H}, 2 \mathrm{CH}_{3}\right), 3.23\left(\mathrm{~s}, 6 \mathrm{H}, 2 \mathrm{CH}_{3}\right), 3.14(\mathrm{q}, 4 \mathrm{H}, J=7.3$ $\left.\mathrm{Hz}, \mathrm{CH}_{2} \mathrm{CH}_{3}\right), 2.41$ (q, 4H, J=5.1 Hz, $\left.\mathrm{CH}_{2}\right), 2.23(\mathrm{~s}, 2 \mathrm{H}$, $\mathrm{CH}_{2}$ ), 1.37 (t, $\left.6 \mathrm{H}, J=7.3 \mathrm{~Hz}, \mathrm{CH}_{2} \mathrm{CH}_{3}\right), 1.07$ (s, 3H, $\mathrm{CH}_{3}$ ), 1.01 (s, 3H, $\left.\mathrm{CH}_{3}\right) ;{ }^{13} \mathrm{C}$ NMR $\left(100 \mathrm{MHz} \mathrm{CDCl}_{3}\right): \delta=199.0$, $180.5,165.3,164.3,152.5,149.7,136.8,131.5,129.9,126.5$, 124.2, 115.5, 114.7, 89.9, 50.9, 45.5, 41.7, 31.3, 30.7, 28.2, 11.1; LC/MS (ESI): 507 [M] ${ }^{+}$; Anal. for $\mathrm{C}_{29} \mathrm{H}_{37} \mathrm{~N}_{3} \mathrm{O}_{5}$; calcd: C, 68.62; H, 7.35; N, 8.28; Found: C, 68.65; H, 7.34; N, 8.30.

\section{5-((2-Hydroxy-4,4-dimethyl-6-oxocyclohex-1-en-1-yl) (4-hydroxyphenyl)methyl)-1,3-dimethyl-2,6-dioxo-1,2,3, 6-tetrahydropyrimidin-4-olate (4 k)}

$4 \mathbf{k}$ was prepared from 1,3-dimethylbarbituric acid 1a, dimedone $\mathbf{2}$ and $p$-hydroxybenzaldehyde $\mathbf{3}$ according to the general procedure (GP1) yielding a white solid material (645 mg, $1.36 \mathrm{mmol}, 91 \%)$ m.p: $162^{\circ} \mathrm{C}$; IR $\left(\mathrm{KBr}, \mathrm{cm}^{-1}\right)$ : 23097, 2939, 2884, 2828, 2498, 1747, 1574, 1530, 1506, 1466, 1384, 1241; ${ }^{1} \mathrm{H}$ NMR (400 MHz, DMSO- $\left.d_{6}\right): \delta 14.52$ (s, 1H, OH), 8.50 (brs, 1H, OH), 6.76 (d, 2H, J= 8.0 Hz, Ph), 6.50 (d, 2H, J=8.0 Hz, Ph), 6.04 (s, $1 \mathrm{H}$, benzyl-H), 3.07 (s, $12 \mathrm{H}, 2 \mathrm{CH}_{3}$ ), 3.14 (q, 4H, J=7.3 Hz, $\mathrm{CH}_{2} \mathrm{CH}_{3}$ ), 2.92 (q, 4H, J= $\left.13.9 \mathrm{~Hz}, \mathrm{CH}_{2}\right), 206\left(\mathrm{~s}, 4 \mathrm{H}, \mathrm{CH}_{2}\right), 1.12\left(\mathrm{t}, 6 \mathrm{H}, J=7.3 \mathrm{~Hz}, \mathrm{CH}_{2} \mathrm{CH}_{3}\right)$, 0.98 (s, $\left.3 \mathrm{H}, \mathrm{CH}_{3}\right) ;{ }^{13} \mathrm{C}$ NMR (100 MHz, DMSO- $\left.d_{6}\right): \delta=198.0$, 188.5, 154.1, 136.6, 128.3, 115.3, 114.3, 90.1, 50.9, 45.5, 42.1, 31.6, 30.7, 29.7, 11.7; LC/MS (ESI): 473 [M] ${ }^{+}$; Anal. for $\mathrm{C}_{25} \mathrm{H}_{35} \mathrm{~N}_{3} \mathrm{O}_{6}$; calcd: C, 63.41; H, 7.45; N, 8.87; Found: C, 63.40; H, 7.43; N, 8.85.

5-((2-Hydroxy-4,4-dimethyl-6-oxocyclohex-1-en-1-yl)(phenyl) methyl)-2,6-dioxo-1,2,3,6-tetrahydropyrimidin-4-olate (4 I) $\mathbf{4} \mathbf{~ m}$ was prepared from barbituric acid $\mathbf{1 b}$, dimedone $\mathbf{2}$ and benzaldehyde $\mathbf{3}$ according to the general procedure 

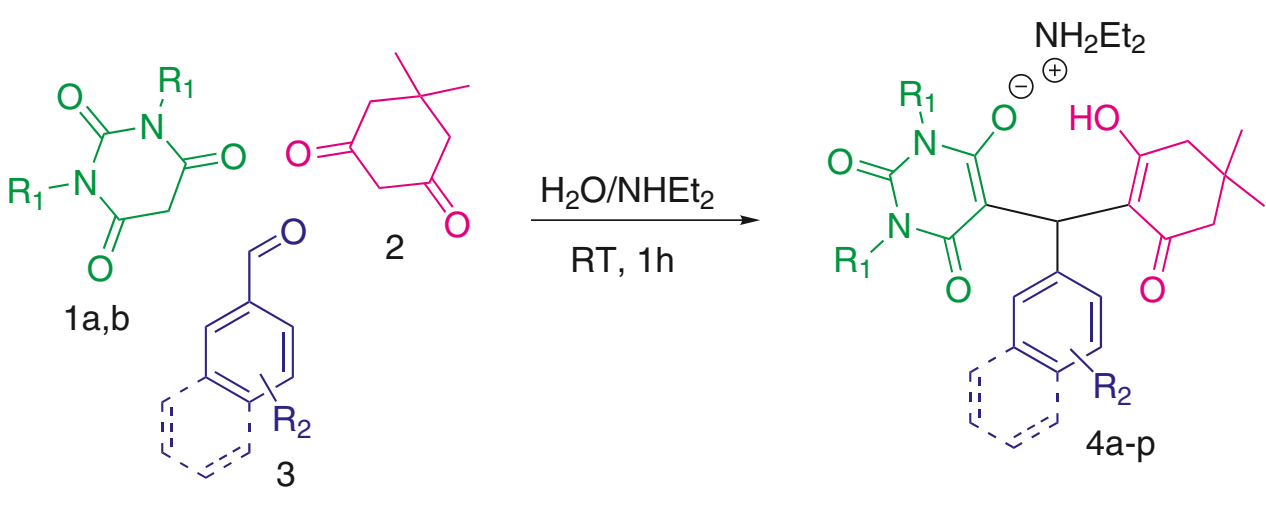

Scheme 2 Synthesis of $4 a-p$.

(GP1) yielding a white solid material (598 mg, $1.39 \mathrm{mmol}$, 93\%). m.p: $215^{\circ} \mathrm{C}$; IR (KBr, $\mathrm{cm}^{-1}$ ): 3027, 2948, 2867, 2156, $1683,1593,1451,1374,1291,1257,1141^{1} \mathrm{H}-\mathrm{NMR}$ $\left(400 \mathrm{MHz}, \mathrm{CDCl}_{3}\right): \delta 12.26(\mathrm{~s}, 1 \mathrm{H}, \mathrm{OH}), 9.31$ (brs, $2 \mathrm{H}$, $\mathrm{NH}), 7.12$ (m, 5H, Ph), 5.52 (s, 1H, benzyl-H), 2.99 (q, 4H, $\left.J=7.3 \mathrm{~Hz}, \mathrm{CH}_{2} \mathrm{CH}_{3}\right), 2.45\left(\mathrm{~d}, 4 \mathrm{H}, J=5.1 \mathrm{~Hz}, \mathrm{CH}_{2}\right), 1.24$ (t, 6H, J= 7.3 Hz, $\left.\mathrm{CH}_{2} \mathrm{CH}_{3}\right), 1.09\left(\mathrm{~s}, 3 \mathrm{H}, \mathrm{CH}_{3}\right), 1.03(\mathrm{~s}, 3 \mathrm{H}$, $\left.\mathrm{CH}_{3}\right) ;{ }^{13} \mathrm{C} \mathrm{NMR}\left(100 \mathrm{MHz}, \mathrm{CDCl}_{3}\right): \delta=198.5,180.8$, $152.5,142.5,128.0,126.7,125.1,116.3,90.9,51.4,45.9$, 42.2, 33.0, 28.4, 27.6, 11.3; LC/MS (ESI): $429\left[\mathrm{M}^{+}\right.$; Anal. for $\mathrm{C}_{23} \mathrm{H}_{31} \mathrm{~N}_{3} \mathrm{O}_{5}$; calcd: C, 64.32; H, 7.27; N, 9.78; Found: C, 64.29; H, 7.29; N, 9.80.

5-((2-Hydroxy-4,4-dimethyl-6-oxocyclohex-1-en-1-yl)(p-tolyl) methyl)-2,6-dioxo-1,2,3,6-tetrahydropyrimidin-4-olate (4 m) 4n was prepared from barbituric acid 1a, dimedone 2 and tolualdehyde $\mathbf{3}$ according to the general procedure (GP1) yielding a white solid material (604 mg, $1.36 \mathrm{mmol}, 91 \%)$. m.p: $213^{\circ} \mathrm{C}$; IR $\left(\mathrm{KBr}, \mathrm{cm}^{-1}\right)$ : 3150, 2955, 2867, 1690, 1592, 1508, 1375, 1256, 1232, 1167; ${ }^{1} \mathrm{H}$ NMR (400 MHz, $\left.\mathrm{CDCl}_{3}\right)$ : $\delta 13.31$ (s, 1H, OH), 8.83 (brs, $2 \mathrm{H}, \mathrm{NH}), 7.27$ (d, 2H, $J=$ $8.0 \mathrm{~Hz}, \mathrm{Ph}), 7.00$ (d, $2 \mathrm{H}, J=8.0 \mathrm{~Hz}, \mathrm{Ph}), 5.88$ (s, $1 \mathrm{H}$, benzyl$\mathrm{H}), 2.83\left(\mathrm{q}, 4 \mathrm{H}, J=7.3 \mathrm{~Hz}, \mathrm{CH}_{2} \mathrm{CH}_{3}\right), 2.31(\mathrm{~d}, 4 \mathrm{H}, J=5.1 \mathrm{~Hz}$, $\mathrm{CH}_{2}$ ), $2.23\left(\mathrm{~s}, 3 \mathrm{H}, \mathrm{CH}_{3}\right), 1.19\left(\mathrm{t}, 6 \mathrm{H}, J=7.3 \mathrm{~Hz}, \mathrm{CH}_{2} \mathrm{CH}_{3}\right.$ ), $1.04\left(\mathrm{~s}, 3 \mathrm{H}, \mathrm{CH}_{3}\right), 1.02\left(\mathrm{~s}, 3 \mathrm{H}, \mathrm{CH}_{3}\right) ;{ }^{13} \mathrm{C} \mathrm{NMR}(100 \mathrm{MHz}$, $\left.\mathrm{CDCl}_{3}\right): \delta=196.5,180.1,152.8,140.5,131.4,130.7,128.7$, 128.6, 118.5, 115.6, 91.0, 50.9, 42.8, 31.6, 31.5, 29.2, 28.3, 27.8, 20.9, 11.3; LC/MS (ESI): $443[\mathrm{M}]^{+}$; Anal. for $\mathrm{C}_{24} \mathrm{H}_{33} \mathrm{~N}_{3} \mathrm{O}_{5}$; calcd: C, 64.99; H, 7.50; N, 9.47; Found: C, 64.95; H, 7.49; N, 9.50.

\section{5-((4-Chlorophenyl)(2-hydroxy-4,4-dimethyl-6-oxocyclohex-} 1-en-1-yl)methyl)-2,6-dioxo-1,2,3,6-tetrahydropyrimidin-4olate (4n)

4o was prepared from barbituric acid 1a, dimedone $\mathbf{2}$ and $p$-chlorobenzaldehyde 3 according to the general procedure (GP1) yielding an oily product (625 $\mathrm{mg}, 1.35 \mathrm{mmol}, 90 \%)$. IR $\left(\mathrm{KBr}, \mathrm{cm}^{-1}\right)$ : 3049, 2954, 2865, 2499, 1738, 1699, 1590, 1483, 1375, 1292, 1258, 1225, 1205; ${ }^{1} \mathrm{H}$ NMR (400 MHz,
$\mathrm{CDCl}_{3} \delta 13.32$ (s, 1H, OH), 8.83 (brs, $\left.2 \mathrm{H}, \mathrm{NH}\right), 7.27$ (d, 2H, $J=8.0 \mathrm{~Hz}, \mathrm{Ph}), 7.00(\mathrm{~d}, 2 \mathrm{H}, J=8.0 \mathrm{~Hz}, \mathrm{Ph}), 5.89$ (s, $1 \mathrm{H}$, benzyl-H), 2.88 (q, 4H, J=7.3 Hz, $\left.\mathrm{CH}_{2} \mathrm{CH}_{3}\right), 2.31$ (d, $4 \mathrm{H}$, $J=5.1 \mathrm{~Hz}, \mathrm{CH}_{2}$ ), 1.19 (t, 6H, J=7.3 Hz, $\mathrm{CH}_{2} \mathrm{CH}_{3}$ ), 1.09 $\left(\mathrm{s}, 3 \mathrm{H}, \mathrm{CH}_{3}\right), 1.03\left(\mathrm{~s}, 3 \mathrm{H}, \mathrm{CH}_{3}\right) ;{ }^{13} \mathrm{C} \mathrm{NMR}(100 \mathrm{MHz}$, $\left.\mathrm{CDCl}_{3}\right): \delta=190.9,141.0,134.8,131.0,129.5,128.3,115.3$, 91.1, 47.1, 42.7, 31.6, 31.5, 29.1, 28.2, 27.8, 11.3; LC/MS (ESI): $463[\mathrm{M}]^{+}$; Anal. for $\mathrm{C}_{23} \mathrm{H}_{30} \mathrm{ClN}_{3} \mathrm{O}_{5}$; calcd: C, 59.54; H, 6.52; Cl, 7.64; N, 9.06; Found: C, 59.57; H, 6.51; Cl, 7.60; $\mathrm{N}, 9.02$.

\section{5-((4-Bromophenyl)(2-hydroxy-4,4-dimethyl-6-oxocyclohex- 1-en-1-yl)methyl)-2,6-dioxo-1,2,3,6-tetrahydropyrimidin- 4-olate (40)}

4n was prepared from barbituric acid 1a, dimedone 2 and $p$-bromobenzaldehyde $\mathbf{3}$ according to the general procedure (GP1) yielding a white solid material $(678 \mathrm{mg}, 1.33 \mathrm{mmol}$, 89\%). m.p: $208^{\circ} \mathrm{C}$; IR (KBr, $\left.\mathrm{cm}^{-1}\right)$ : 3093, 2939, 2885, 2829, 2551, 1746, 1686, 1576, 1506, 1466, 1416, 1268, 1241; ${ }^{1} \mathrm{H}$ NMR (400 MHz, $\mathrm{CDCl}_{3}$ ): $\delta 13.31$ (s, 1H, OH), 8.67 (brs, 2H, NH), 7.05 (m, 4H, Ph), 5.79 (s, 1H, benzyl-H), 2.79 (q, $\left.4 \mathrm{H}, J=7.3 \mathrm{~Hz}, \mathrm{CH}_{2} \mathrm{CH}_{3}\right), 2.35\left(\mathrm{~d}, 4 \mathrm{H}, J=5.1 \mathrm{~Hz}, \mathrm{CH}_{2}\right.$ ), $1.21\left(\mathrm{t}, 6 \mathrm{H}, J=7.3 \mathrm{~Hz}, \mathrm{CH}_{2} \mathrm{CH}_{3}\right), 1.11\left(\mathrm{~s}, 3 \mathrm{H}, \mathrm{CH}_{3}\right), 1.03$ $\left(\mathrm{s}, 3 \mathrm{H}, \mathrm{CH}_{3}\right) ;{ }^{13} \mathrm{C} \mathrm{NMR}\left(100 \mathrm{MHz}, \mathrm{CDCl}_{3}\right): \delta=198.5$, $180.1,152.8,140.5,131.4,130.7,128.7,128.6,118.5,115.6$, 91.0, 50.9, 42.8, 31.6, 31.5, 29.2, 28.3, 27.8, 11.3; LC/MS (ESI): $508[\mathrm{M}]^{+}$; Anal. for $\mathrm{C}_{23} \mathrm{H}_{30} \mathrm{BrN}_{3} \mathrm{O}_{5}$; calcd: C, 54.34; $\mathrm{H}, 5.95$; Br, 15.72; N, 8.27; Found: C, 54.35; H, 5.96; Br, $15.69 ; \mathrm{N}, 8.30$.

\section{5-((2-Hydroxy-4,4-dimethyl-6-oxocyclohex-1-en-1-yl) (naphthalen-2-yl)methyl)-2,6-dioxo-1,2,3,6- tetrahydropyrimidin-4-olate (4p)}

$\mathbf{4 q}$ was prepared from barbituric acid 1a, dimedone $\mathbf{2}$ and 2-naphthaldehyde $\mathbf{3}$ according to the general procedure (GP1) yielding an oily product (646 $\mathrm{mg}, 1.35 \mathrm{mmol}, 90 \%)$. IR $\left(\mathrm{KBr}, \mathrm{cm}^{-1}\right)$ : 3049, 2948, 2863, 2725, 1685, 1594, 1508, $1371,1252,1216 ;{ }^{1} \mathrm{H}$ NMR $\left(400 \mathrm{MHz}, \mathrm{CDCl}_{3}\right): \delta 14.25$ (s, 1H, OH), 7.46-7.22 (m, 7H, naphthyl), $6.21(\mathrm{~s}, 1 \mathrm{H}$, 
benzyl-H), $3.27\left(\mathrm{~s}, 6 \mathrm{H}, 2 \mathrm{CH}_{3}\right), 3.25\left(\mathrm{~s}, 6 \mathrm{H}, 2 \mathrm{CH}_{3}\right), 3.14$ (q, $4 \mathrm{H}, J=7.3 \mathrm{~Hz}, \mathrm{CH}_{2} \mathrm{CH}_{3}$ ), 2.41 (q, $4 \mathrm{H}, J=5.1 \mathrm{~Hz}, \mathrm{CH}_{2}$ ), $2.23\left(\mathrm{~s}, 2 \mathrm{H}, \mathrm{CH}_{2}\right), 1.37\left(\mathrm{t}, 6 \mathrm{H}, J=7.3 \mathrm{~Hz}, \mathrm{CH}_{2} \mathrm{CH}_{3}\right), 1.07$ $\left(\mathrm{s}, 3 \mathrm{H}, \mathrm{CH}_{3}\right), 1.01\left(\mathrm{~s}, 3 \mathrm{H}, \mathrm{CH}_{3}\right) ;{ }^{13} \mathrm{C}$ NMR $(100 \mathrm{MHz}$, $\left.\mathrm{CDCl}_{3}\right): \delta=199.1,180.5,165.5,164.2,152.5,149.7,136.8$, $131.5,129.9,126.5,124.2,115.5,114.7,89.9,50.9,45.5$, 41.7, 31.3, 30.7, 28.2, 11.3; LC/MS (ESI): $479[\mathrm{M}]^{+}$; Anal. for $\mathrm{C}_{27} \mathrm{H}_{33} \mathrm{~N}_{3} \mathrm{O}_{5}$; calcd: C, 67.62; H, 6.94; N, 8.76; Found: C, 67.65; H, 6.96; N, 8.80.

\section{Competing interests}

The authors declare that they have no competing interests.

\section{Authors' contributions}

$A B$ proposed the subject, designed the study. AMA carried out the synthesis of all the products. YNM and AMA helped in the results and discussion. MRHS carried out NMR spectroscopy and elemental analysis. HG and HKF carried out the X-ray crystallography part. AB prepared draft the manuscript. All the authors read and approved the final manuscript.

\section{Acknowledgements}

The authors extend their appreciation to the Deanship of Scientific Research at King Saud University for funding the work through the research group project Number RGP- VPP- 257.

\section{Author details}

'Department of Chemistry, College of Science, King Saud University, P.O. Box 2455, Riyadh 11451, Saudi Arabia. ${ }^{2}$ Department of Chemistry, Faculty of Science, Alexandria University, P.O. Box 426, Ibrahimia, Alexandria 21321, Egypt. ${ }^{3}$ Department of Pharmaceutical Chemistry, College of Pharmacy, King Saud University, P.O. Box 2457, Riyadh 11451, Saudi Arabia.

Received: 27 October 2013 Accepted: 28 January 2014 Published: 1 February 2014

\section{References}

1. Grieco PA: Organic Synthesis in Water. London: Thomson Science; 1998:1-278.

2. Li CJ: Organic reactions in aqueous media with a focus on carbon-carbon bond formations: a decade update. Chem Rev 2005, 105:3095-3165.

3. Bojarski JT, Mokrocz JL, Barton HJ, Paluchowska MH: Recent progress in barbituric acid chemistry. Adv Heterocycl Chem 1985, 38:229-297.

4. Undheim K, Bennecke T, Katritzky AR, Rees CW, Scriven EFV, Boulton AJ: (Hrsg.): Comprehensive Heterocyclic Chemistry, Volume 6. Oxford: Elsevier Pergamon; 1996. Suppl. 93.

5. Angerer S: Product class 12: pyrimidines. Sci Synth 2004, 16:379-572.

6. Sans SRG, Chosaz MG: Historical aspects and applications of barbituric acid derivatives. Pharmazie 1988, 43:827-829.

7. Taylor JB: Modern Medical Chemistry. New York: Prentice Hall; 1994.

8. Guerin DJ, Mazeas D, Musale MS, Naguib FNM, Safarjalani ONA, Kouni MH, Panzica RP: Uridine phosphorylase inhibitors: chemical modification of benzyloxybenzyl barbituric acid and its effects on UrdPase inhibition. Bioorg Med Chem Lett 1999, 9:1477-1480.

9. Andrews G: Medical pharmacology. Saint Louis, MO: The CV Mosby Co; 1976:243-250.

10. Foye WO: Principles of medicinal chemistry. Pennsylvania, PA: Lea \& Febiger; 1989:143-237.

11. Goodman LS, Gilman A: The pharmacological basis of therapeutics. New Delhi: Mc Graw-Hill; 1991:358-360.

12. Senda S, Izumi H, Fujimura H: Uracil derivatives and related compounds. VI. Derivatives of 5-alkyl-2,4,6-trioxoperhydropyrimidine as anti-inflammatory agents. Arznein Forsch 1967, 17:1519-1523.

13. Fisher E, Moring JR: Ueber eine neue klasse von schlafmitteln. Ther Ggw 1903, 44:97-105

14. Doran WJ: Barbituric acid hypnotics. Med Chem 1959, 4:164-167.

15. Bobranski B: Progress in chemistry of barbituric acid. Wiad Chem 1977, 31:231-278.

16. Anderson GL, Shim JL, Brown AD: Pyrido[2,3-d]pyrimidines. IV. Synthetic studies leading to various oxopyrido[2,3-d]pyrimidines. J Org Chem 1976, 41:1095-1099.
17. Grivaky EM, Lee $S$, Siyal CW, Duch DS, Nichol CA: Synthesis and antitumor activity of 2,4-diamino-6-(2,5-dimethoxybenzyl)-5-methylpyrido[2,3-d] pyrimidine. J Med Chem 1980, 23:327-329.

18. El Ashry El SH, Awada LF, El Kilany Y, El Ibrahim S: Chapter 1 dimedone: a versatile precursor for annulated heterocycles. Adv Heterocyd Chem 2009, 98:1-141.

19. Saitoh T, Taguchi K, Hiraide M: Derivatization of formaldehyde with dimedone and subsequent sorption on sodium dodecylsulfate/alumina admicelles for fluorometric analysi. Anal Sci 2002, 18:1267-1268.

20. Cremlyn RJ, Saunders D: Chlorosulfonation of 9-aryl 3,36,6-tetramethyloctahydroxanthen-1,8-diones. Phosphorus Sulfur Silicon 1993, 81:73-82.

21. Bodini ME, Pardo J, Arancibia V: Spectrophotometric determination of selenium(IV) with 5,5-dimethyl-1,3-cyclohexanedione. Talanta 1990, 37:439-442.

22. Gruttadauria M, Giacalone F, Marculesco AM, Meo PL, Riela S, Noto R Hydrophobically directed aldol reactions: polystyrene-supported L-proline asa recyclable catalyst for direct asymmetric Aldol reactions in the presence of water. Eur J Org Chem 2007, 2007:4688.

23. Breslow R: Determining the geometries of transition states by use of antihydrophobic additives in water. Acc Chem Res 2004, 37:471-478.

24. Blackmond DG, Armstrong A, Coombe V, Wells A: Water in organocatalytic processes: Debunking the Myths. Angew Chem Int Ed Engl 2007, 46:3798-3800.

25. Abaee MS, Cheraghi S, Navidipoor S, Mojtahedi MM, Forghani S: An efficient tandem aldol condensation-thia-Michael addition process. Tetrahedron Lett 2012, 53:4405-4408.

26. Barakat A, Al-Majid AA: Shahidul Islam M, Al-Othman ZA: Highly enantioselective Friedel - Crafts alkylations of indoles with $a$, $\beta$-unsaturated ketones under $\mathrm{Cu}(\mathrm{II})$-simple oxazoline-imidazoline catalysts. Tetrahedron 2013, 69:5185-5192.

27. Jursic BS: Preparation of unsubstituted dipyridine-dibarbituric acid ylide through Dimerization of pyridin-2-ylmethylenepyrimidinetrione as a reactive intermediate. J Heterocyclic Chem 2003, 40:167-170.

28. Jursic BS, Neumann DM: Preparation of 5,5'-pyrilidene and 5,5'-quinolidene bis-barbituric acid derivatives. J Heterocyclic Chem 2003, 40:465-474.

29. Barakat A, Al-Majid AM, Al-Najjar HJ, Mabkhot YN, Ghabbour HA, Fun H-K: An efficient and green procedure for synthesis of rhodanine derivatives by Aldol-thia-Michael protocol using aqueous diethylamine. RSC Adv 2014, 4:4909-4916.

30. Al-Najjar HJ, Barakat A, Al-Majid AM, Mabkhot YN, Weber M, Ghabbour HA, Fun H-K: A greener and efficient approach to Michael addition of barbituric acid to nitroalkene in aqueous diethylamine medium. Molecules 2014, 19:1150-1162.

31. Al-Majid AM, Barakat A, Al-Najjar HJ, Mabkhot YN, Ghabbour HA, Fun H-K: Tandem Aldol-Michael reactions in aqueous diethylamine medium: a greener and efficient approach to bis-pyrimidine derivatives. Int J Mol Sci 2013, 14:23762-23773.

doi:10.1186/1752-153X-8-9

Cite this article as: Barakat et al.: Tandem Aldol-Michael reactions in aqueous diethylamine medium: a greener and efficient approach to dimedone-barbituric acid derivatives. Chemistry Central Journal 2014 8:9.

\section{Publish with ChemistryCentral and every scientist can read your work free of charge \\ "Open access provides opportunities to our colleagues in other parts of the globe, by allowing anyone to view the content free of charge." W. Jeffery Hurst, The Hershey Company.}

- available free of charge to the entire scientific community

- peer reviewed and published immediately upon acceptance

- cited in PubMed and archived on PubMed Central

- yours - you keep the copyright

Submit your manuscript here:

http://www.chemistrycentral.com/manuscript/ 